\title{
HOLOPROSENCEFALIA: A PROPOSITO DE UN CASO
}

\author{
Hans Carbonel-Ramirez ${ }^{1, a}$, Jhon Fernández-Roman ${ }^{1, a}$, Jorge Ybaseta-Medina, ${ }^{1, b}$,Ana Kuroki-Ishii ${ }^{2, b}$ \\ Audias Pérez-Aguirre ${ }^{1, b}$
}

1 Hospital Santa María del Socorro. Ica, Perú.

2 Facultad de Medicina, Universidad Nacional San Luis Gonzaga. Ica, Perú.

a Interno de medicina, b Medico Gineco-Obstetra

\section{RESUMEN}

Se reporta el caso de una mujer de 22 años de edad, sin antecedentes de importancia. Fue admitida en el hospital, en pródromos de trabajo de parto, gestación de 40 semanas y malformación cerebral: holoprosencefalia semilobar, hipotelorismo, hidronefrosis derecha leve (diagnóstico ecográfico). Es intervenida mediante la operación cesárea obteniéndose un recién nacido con características de holoprosencefalia siendo lo resaltante el hipotelorismo y la arrinencefalia. No presenta llanto al nacimiento, por lo que se le realiza maniobras de reanimación cardio pulmonar, continùa en mal estado general con periodos de apnea, baja saturación, expansibilidad torácica disminuida, se procede a colocación de tubo endotraqueal, administración de adrenalina, es llevada al servicio de unidad de cuidados intensivos pediátricos, presentando en el trayecto dos episodios de paro cardiaco, el menor luego es colocado en ventilador mecánico, no teniendo una buena evolución ,fallece el día siguiente de nacido.

Palabras Clave: Holoprosencefalia, anomalías del ojo, anomalías congénitas (fuente: DeCS BIREME)

\section{HOLOPROSENCEPHALY: REPORT A CASE}

\begin{abstract}
It is reported the case of a woman, 22 years of age, without important background information. She was admitted to the hospital, in premonitory signs of labor with a gestation of 40 weeks and cerebral malformation: semilobar holoprosencephaly, hipotelorismo, mild right hydronephrosis (sonographic diagnosis). She is operated by the cesarean section obtaining a newborn with characteristics of holoprosencephaly remains what the striking hipotelorismo and arrinencefalia . There is no crying at birth, by what is realized maneuvers cardio pulmonary resuscitation ,continues in poor condition general ,with periods of apnea, low saturation ,chest diminished expansibility, so proceeded to endotracheal tube placement, placement of adrenaline, is taken to the pediatric intensive care units, presenting in the journey two episodes of cardiac arrest ,the minor then is placed on mechanical ventilator ,not taking a good evolution, dies the next day of birth.
\end{abstract}

Key words: Holoprosencephaly, Eye abnormalities, congenital abnormalities (source: MeSH NLM)

\section{INTRODUCCIÓN}

Al comienzo de la tercera semana de vida embrionaria, se empieza a formar el sistema nervioso central, es ahí cuando aparece la placa neural, la cual posteriormente formará el tubo neural que dará origen a estructuras como el prosencéfalo, el mesencéfalo y el romboencéfalo. Cualquier alteración que ocurra en estos importantes períodos traerá consigo consecuencias neurológicas importantes para este futuro $\operatorname{ser}^{(1,2)}$.

Diferentes grados de dismorfismo facial se asocian comúnmente con esta patología, incluyendo ciclopia, etmocefalia, cebocefalia, labio leporino medio y otras alteraciones menos severas. Otros términos tales como arrinencefalia, enfatizando la ausencia de estructuras olfatorias comúnmente encontrada en estos casos, y holotelencefalia, han sido utilizados para denominar esta condición ${ }^{(3)}$.
La etiología de esta malformación es heterogénea y solamente se encuentra la causa entre el $15 \%$ y el $20 \%$ de los casos. Las causas identificadas son: monogénicas, cromosómicas y teratogénicas. Entre los patrones de herencia monogénicos no sindrómicos se encuentran más comúnmente los dominantes con penetrancia incompleta y expresividad variable y menos común los de transmisión recesiva y ligados al cromosoma $\mathrm{X}$. Igualmente, la diabetes materna es el factor teratogénico más importante. El consumo de etanol, ácido retinoico y salicilatos durante el primer trimestre del embarazo también han sido reportados y la alteración cromosómica numérica más frecuentemente relacionada es la trisomía $13^{(4)}$.

Por tratarse de una patología infrecuente y rara en nuestro medio es motivo del reporte. 


\section{CASO CLINIICO}

Paciente de 22 años de edad, con una edad gestacional de 40 semanas, controlada en un puesto de salud, procedente del departamento de Piura, conviviente, ama de casa y con secundaria completa, sin antecedentes perinatales de importancia. Se interna en nuestro hospital, con diagnóstico de pródromo de trabajo de parto, primigesta, refiriendo dolor abdominal tipo contracción uterina. Niega exposición a teratógenos, grupo sanguíneo $\mathrm{O}$, factor $\mathrm{RH}$ $(+)$, glicemia dentro de los valores normales, VDRL negativo, VIH negativo, examen de orina negativo, con 11 controles obstétricos, primer control a partir de la semana 15 , test no estresante: reactivo, controles ecográficos del tercer trimestre donde se informa gestación única de 30 semanas, con malformación cerebral a descartar: holoprosencefalia semilobar, hipotelorismo, feto vivo, hidronefrosis derecha leve fetal (Figura 1,2 y 3 ).

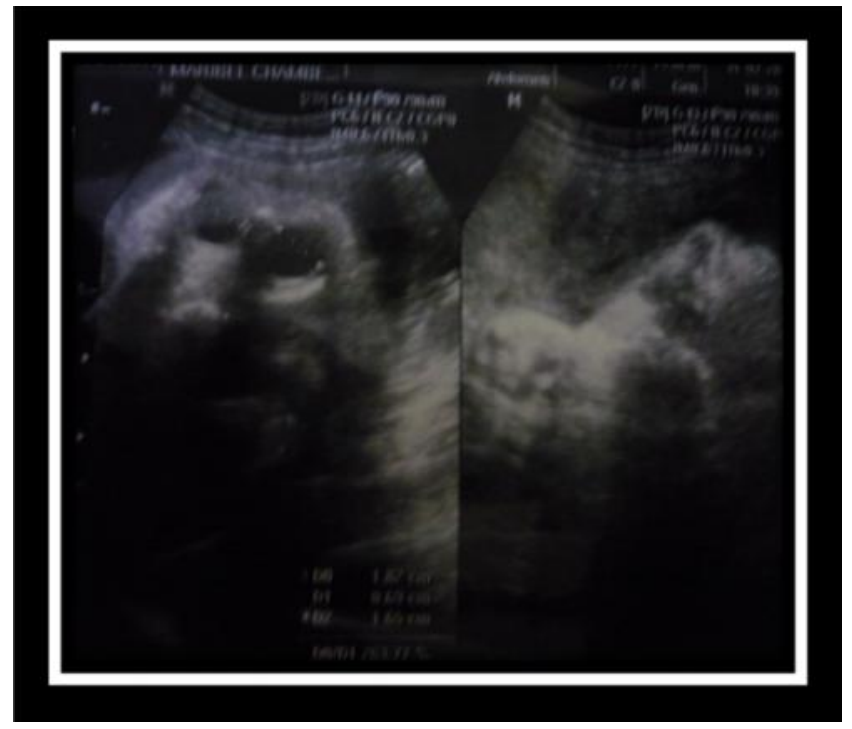

Figura 1. Imágenes ecográficas.a: se aprecia orbitas juntas, b: fosas nasal única

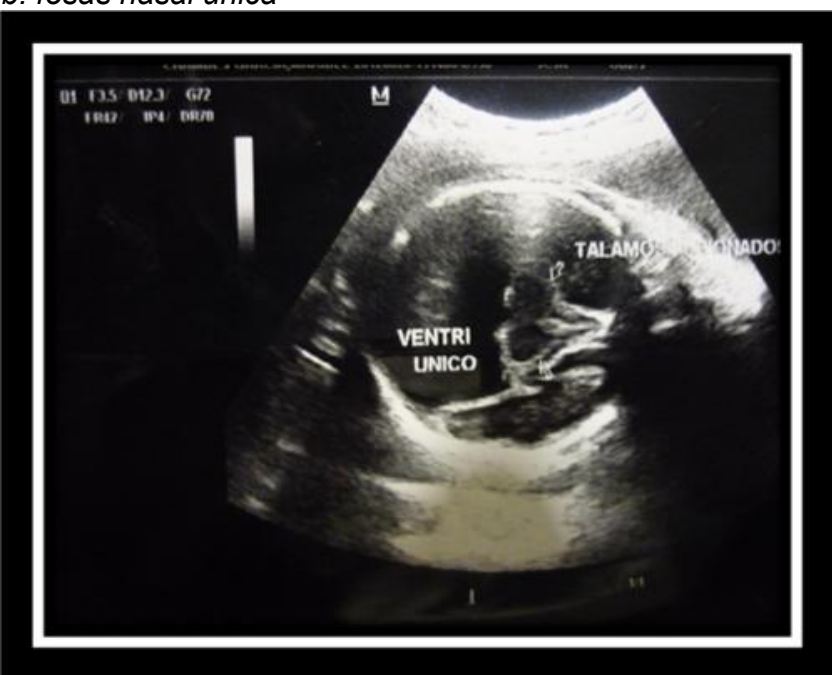

Figura 2. Cavidad ventricular única, fusión de los tálamos, ausencia del septum pellucidum.

La exploración física reveló una presión arterial de 90/60 $\mathrm{mmHg}$, una frecuencia cardiaca de 78, una frecuencia respiratoria de 16 , afebril y a la evaluación por órganos y aparatos no se evidenció alteraciones.

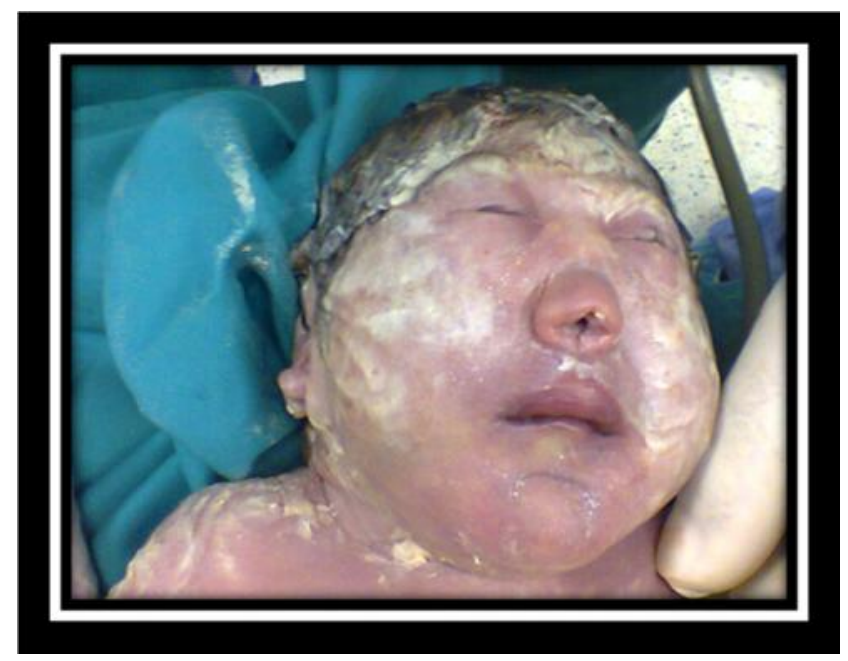

Figura 3. Hipotelorismo, implantación baja del pabellón auricular

El examen obstétrico revelo un abdomen con útero grávido a tres centímetros por debajo del apéndice xifoides, feto en posición longitudinal cefálico derecho, altura uterina de 31 centímetros, frecuencia cardiaca fetal de 128 a 132 por minuto. Peso ponderado fetal de tres kilogramos, una dinámica uterina de $3 / 10^{\prime}+++$; al tacto vaginal se evidencio una dilatación de 0 centímetros, incorporación de 0\% APF 3 , membranas amnióticas integras y pelvis ginecoide.

Presento un hematocrito preparto de $36 \%$ y una hemoglobina de $12 \mathrm{gr} / \mathrm{dl}$, la paciente es hospitalizada en nuestro servicio, fue programada al siguiente día para que sea intervenida quirúrgicamente, realizándose unacesárea segmentaria transversa.

Se obtuvo un producto de sexo femenino, no presentó llanto al nacimiento, por lo que se le realiza maniobras de reanimación cardiopulmonar, continùa en mal estado general, con periodos de apnea, baja saturación, expansibilidad torácica disminuida, por lo que se procede a colocación de tubo endotraqueal, es llevada a la unidad de cuidados intensivos pediátricas, presentando en el trayecto dos episodios de paro cardiaco.

El recién nacido tuvo un peso de 2790 gramos, una talla de 49 centímetros, Apgar al minuto de tres, a los cinco minutos de cinco y a los diez minutos de siete, al examen físico de piel y mucosas: tibia no ictérica, se aprecia malformación congénita en fosa nasal (un solo orificio nasal) y en labio superior (figura.3); aparato respiratorio: murmullo vesicular pasa bien en ambos campos pulmonares, polipnea 
moderada, no tiraje ni retracción xifoidea; aparato cardiovascular: ruidos cardiacos rítmicos, no soplos aparato abdominal: blando depresible, no visceromegalia; aparato genital: sin alteraciones, sexo femenino, teniendo como diagnósticos en la unidad de cuidados intensivos de: recién nacido a término, edad gestacional de 37 semanas, adecuado para la edad gestacional, malformaciones congénitas faciales, holoprosencefalia, cromosomopatías, a descartar sepsis neonatal temprana. El menor luego es colocado en ventilador mecánico, no teniendo una buena evolución, fallece el día siguiente de nacido.

\section{DISCUSIÓN}

Holoprosencefalia (HPE) es una de las anomalías cerebrales más graves causadas por la falta de escisión del prosencéfalo durante la etapa temprana de desarrollo. Más de 200 casos de HPE en embriones humanos se han descrito en la colección de la universidad de Kyoto, donde se realizó el estudio macroscópica e histológicamente, con especial énfasis en las anomalías del cerebro, la cara y los ojos $^{(5)}$.

La no segmentación del prosencéfalo en los dos hemisferios dará como resultado una patología conocida como Holoprosencefalia, la cual se caracteriza por defectos múltiples en la línea media de la cara. Se estima que afecta a 1 de cada 5.000-10.000 nacidos vivos, aunque su incidencia aumenta considerablemente en los embarazos que no llegan a término, siendo su frecuencia hasta de 1 de cada 200-250 fetos. Se debe tener en cuenta que tan sólo el $38 \%$ de los fetos diagnosticados de Holoprosencefalia sobreviven al parto ${ }^{(6)}$.

La clasificación más comúnmente utilizada, aun hoy en día, es la propuesta por DeMyer y Zeman en 1963, la cual establece un espectro de severidad decreciente en la división normal de las estructuras mencionadas. Así, se establece la holoprosencefalia alobar, semilobar y lobar ${ }^{(3)}$. La holoprosencefalia alobar, la más severa de ellas, se caracteriza por la ausencia de la cisura interhemisférica y la hoz del cerebro, hay un ventrículo único primitivo (holoventrículo), los tálamos se encuentran fusionados en la línea media $y$ hay ausencia del tercer ventrículo, neurohipófisis y bulbos olfatorios. En la variedad semilobar, los dos hemisferios cerebrales están parcialmente separados posteriormente, pero hay todavía una cavidad ventricular única. Tanto en la forma alobar como en la semilobar, el techo de la cavidad ventricular, la tela coroidea, normalmente envuelta dentro del cerebro, puede protruir entre la convexidad cerebral y el cráneo, formando un quiste de tamaño variable, el saco dorsal. Las holoprosencefalias alobares y semilobares a menudo se asocian con microcefalia $\mathrm{o}$, menos frecuentemente, con macrocefalia la que invariablemente ocurre por hidrocefalia obstructiva. Por último, la variedad lobar se caracteriza por la presencia de una cisura interhemisférica bien desarrollada tanto anterior como posteriormente, pero se mantiene cierto grado de fusión de los gyrus cingulados del cavum y septum pellucidum.
El ultrasonido tiene un gran valor para el diagnóstico de la Holoprosencefalia, siendo la clave del mismo la demostración del ventrículo único. Adicionalmente la identificación de anormalidades faciales puede añadir confiabilidad al diagnóstico ${ }^{(8)}$, el manejo de estos pacientes debe ser conservador y poco agresivo ya que la mayoría de estos pacientes tienden a morir después del nacimiento, explicándosele a la familia de la severidad y el mal pronóstico que tienen de poder sobrevivir.

Fuentes de financiamiento: autofinanciado.

Conflicto de intereses: los autores declaran no tener conflictos de interés.

\section{Correspondencia:}

Hans Carbonel Ramírez

Correo electrónico: hans_carbonelR@hotmail.com

\section{REFERENCIAS BIBLIOGRÁFICAS}

1. Moore KL, Persaud TVN. Embriología clínica. $8^{\mathrm{a}}$ Ed. Elsevier, 2008.

2. Langman S. Embriología Médica con orientación clínica. $10^{\mathrm{a}}$ ed. Editorial Panamericana, 2007.

3. Haratz-Rubinstein N,Monteagudo A,TimorTritsch IE, Tineo R. Perspectivas en holoprosencefalia. Caracas.1995;103(4):305-15.

4. Saldarriaga W. Vigilancia epidemiológica de ciclopía en el Hospital Universitario del Valle, Cali (Colombia) 2004-2008. Rev Colomb Obstet Ginecol. 2010; 61(1): 12-17.

5. Yamada S. Embryonic holoprosencephaly: pathology and phenotypic variability. Congenit Anom (Kyoto) 2006, 46(4):164-171.

6. Guevara LC. Reporte de un caso clínico de Holoprosencefalia. CIMEL. 2006; 11(2): 100-104.

7. Sánchez J, Carstens E, Gutiérrez J, Dezerega V, Sepúlveda W. Holoprosencefalia: diagnóstico prenatal ultrasonográfico y manejo. Rev Chil Ultrasonog 1998;1(4):120-4.

8. Montilla L, Rodríguez A, Chacín B, Romero L. Etmocefalia. Reporte de un caso. Revista de la Facultad de Medicina. 2008; 17 (1): 25-28

Recibido: 11 / 08/2012

Aceptado para publicación: 11 / 11 / 2012 